\title{
Heuristic Machine Learning Feedforward Algorithm for Predicting Shelf Life of Processed Cheese
}

\author{
Sumit Goyal and Gyanendra Kumar Goyal \\ National Dairy Research Institute, Karnal , India \\ E-mail: thesumitgoyal@gmail.com;gkg5878@yahoo.com
}

\begin{abstract}
This paper describes the potential of machine learning feedforward algorithm for predicting shelf life of processed cheese. Soluble nitrogen, $\mathrm{pH}$, Standard plate count, Yeast \& mould count, and Spore count were taken as input parameters, and sensory score as output parameter for developing feedforward single and multilayer models. The dataset was divided into two disjoint sets, one for training and the other for validation. Backpropagation algorithm based on Bayesian Regularization was selected for training the feedforward models. Mean square error, root mean square error, coefficient of determination and nash - sutcliffo coefficient performance measures were used for testing prediction potential of the developed models. The study revealed that feedforward models are good in predicting shelf life of processed cheese.
\end{abstract}

Keywords: ANN, Shelf Life, Prediction, Feedforward, Processed Cheese, Machine Learning, Heuristic

\section{Introduction}

Throughout the years, the computational changes have brought growth to new technologies. Artificial Neural Networks (ANN) over the years has given various solutions to the industry. Designing and implementing intelligent systems has become a crucial factor for the innovation and development of better products for society. Such is the case of the implementation of artificial life as well as giving solution to interrogatives that linear systems are not able to resolve. Among the 
newest signal processing technologies nowadays, ANN is developed with a systematic step-by-step procedure which optimizes a criterion commonly known as the learning rule. Neural network is a parallel system, capable of resolving paradigms that linear computing cannot. An ANN is a system based on the operation of biological neural networks, in other words, is an emulation of biological neural system. The input/output training data in ANNs is fundamental for these networks as it conveys the information which is necessary to discover the optimal operating point. In addition, non linear natures make neural network processing elements a very flexible system. Basically, an ANN is a system that receives an input, process the data, and provides an output [1]. Paper highlights the importance of ANNs based feedforward models for predicting shelf life of processed cheese stored at $30^{\circ} \mathrm{C}$. Processed cheese is very nutritious and manufactured from ripened Cheddar cheese. This variety of cheese has several advantages over raw cheese, such as tastier and longer shelf life. It is a nourishing high protein food, i.e., a welcome supplement to meat protein [2].

\subsection{Feedforward Neural Network}

A feedforward neural network is an ANN where connections between the units do not form a directed cycle. This is different from recurrent neural networks. The feedforward neural network was the first and arguably simplest type of artificial neural network devised. In this network, the information moves in only one direction, forward, from the input nodes, through the hidden nodes (if any) and to the output nodes. There are no cycles or loops in the network [3].

\subsection{Single Layer Feedforward Neural Network}

Single layer perceptron network consists of a single layer of output nodes; the inputs are fed directly to the outputs via a series of weights. In this way it can be considered the simplest kind of feed-forward network. The sum of the products of the weights and the inputs is calculated in each node, and if the value is above some threshold (typically 0 ) the neuron fires and takes the activated value (typically 1); otherwise it takes the deactivated value (typically -1). Neurons with this kind of activation function are also called artificial neurons or linear threshold units. In the literature the term perceptron often refers to networks consisting of just one of these units. A similar neuron was described by Warren McCulloch and Walter Pitts in the 1940s [3].

\subsection{Shelf Life}

Shelf life studies provide important information to product manufacturers and developers to ensure that the consumer will continue to get a high quality product for a significant period of time after its production. Since the shelf life evaluation conducted in the laboratory is a long time-consuming process, and does not fit 
with the speed requirement of the food industry; therefore accelerated methods for estimating the shelf life of food products have been recently developed. The modern food industry has flourished because of its ability to deliver a wide variety of high quality food products to consumers. This has been achieved by building stability into the products through adopting various technological techniques like processing, packaging, and additives. Consumer demands for convenience have fueled new innovations in the food product development, packaging and chemical industries, and the widespread desire for products to use in the microwave oven has added further impetus to this effort. As an increasing number of new foods compete for getting space on supermarket shelves, the words "speed and innovation" have become the watchwords for food companies seeking to become "first to market" with successful products. Overall quality of the product is of prime importance in present day competitive market and needs to be built into the speed and innovation system. How the consumer feels about the product is the ultimate index of food quality. Therefore, the quality built in during the development of the product and production process must last through the distribution and consumption stages [4].

ANNs have been applied for predicting shelf life of milky white dessert jeweled with pistachio [5], Kalakand [6], and instant coffee flavoured sterilized drink [7, 8]. Time-Delay and Linear Layer models have been developed for predicting shelf life of soft mouth melting milk cakes [9]. Elman and self-organizing models determined shelf life of soft cakes [10]. Radial Basis models were developed for predicting shelf life of brown milk cakes decorated with almonds [11], cakes [12]. The growing consumption of low- and reduced-fat dairy products demands routine control of their authenticity by health agencies. The usual analyses of fat in dairy products are very simple laboratory methods; however, they require manipulation and use of reagents of a corrosive nature, such as sulfuric acid, to break the chemical bonds between fat and proteins. Additionally, they generate chemical residues that require an appropriate destination. In this work, the use of an artificial neural network based on simple instrumental analyses, such as $\mathrm{pH}$, color, and hardness (inputs) was proposed for the classification of commercial yogurts in the low- and reduced-fat categories (outputs). A total of 108 strawberry-flavoured yogurts (48 probiotic low-fat, 36 low-fat, and 24 full-fat yogurts) belonging to several commercial brands and from different batches were used in this research. The statistical analysis showed different features for each yogurt category; thus, a database was built and a neural model was trained with the Levenberg-Marquardt algorithm by using the neural network toolbox of the software MATLAB 7.0.1. Validation with unseen data pairs showed that the proposed model was $100 \%$ efficient. Because the instrumental analyses do not require any sample preparation and do not produce any chemical residues, the proposed procedure is a fast and interesting approach to monitoring the authenticity of these products [13]. A rapid and non-destructive method has been devised for the characterization of chocolate samples based on diffuse reflectance near-infrared Fourier transform spectroscopy (DRIFTS) and ANNs. This 
methodology provides a potentially useful alternative to time-consuming chemical methods of analysis. To assess its utility, 36 chocolate samples purchased from the Spanish market were analyzed for the determination of the main nutritional parameters like carbohydrates, fat, proteins, energetic value and cocoa content. Direct triplicate measurements of each sample were carried out by DRIFTS. Cluster hierarchical analysis was used for selecting calibration and validation data sets, resulting in a calibration set comprised of 19 samples and a validation data set of 17 samples. As it is common the presence of non-linear effect in reflectance spectroscopy, ANNs was chosen for data pre-treatment. The root-mean-square error of prediction (RMSEP) values obtained for carbohydrates, fat, energetic value and cocoa were $1.0 \%(\mathrm{w} / \mathrm{w}), 1.0 \%(\mathrm{w} / \mathrm{w}), 50 \mathrm{~kJ}(100 \mathrm{~g})-1$ and $1.4 \%$, respectively. The mean difference $(\mathrm{dx}-\mathrm{y})$ and standard deviation of mean differences $(\mathrm{sx}-\mathrm{y})$ of the carbohydrates, fat, proteins content, energetic value and cocoa content were 0.9 and $2.4 \%(\mathrm{w} / \mathrm{w}), 0.2$ and $1.0 \%(\mathrm{w} / \mathrm{w}), 9.1$ and $50 \mathrm{~kJ}(100$ g) -1 , and -0.5 and $1.4 \%$, respectively. The maximum relative error for the prediction (QC) of any of these parameters for a new sample did not exceed 5.2\% [14]. Radial basis (exact fit) model was suggested for estimating the shelf life of an extremely popular milk based sweetmeat namely burfi. The input variables were the lab data of the product relating to moisture, titratable acidity, free fatty acids, tyrosine, and peroxide value; and the overall acceptability score was output. Mean square error, root mean square error, coefficient of determination and Nash-Sutcliffo coefficient were applied for comparing the prediction ability of the developed models. The observations indicated exceedingly well correlation between the actual data and the predicted values, with a high determination coefficient and Nash-Sutcliffo coefficient establishing that the models were able to analyze non-linear multivariate data with very good performance and shorter calculation time. From the study it was concluded that the developed model, which is very convenient, less expensive and less time consuming, can be a good alternative to expensive, time consuming and cumbersome laboratory testing method for estimating the shelf life of burfi [15]. Changes in the physical, chemical, and microbiological structure of yogurt determine the storage and shelf life of the product. In this study, microbial counts and $\mathrm{pH}$ values of yogurt during storage were determined at $\mathrm{d} 1,7$, and 14. Simultaneously, image processing of yogurt was digitized by using a machine vision system (MVS) to determine color changes during storage, and the obtained data were modeled with an artificial neural network (ANN) for prediction of shelf life of set-type whole-fat and lowfat yogurts. The ANN models were developed using back-propagation networks with a single hidden layer and sigmoid activation functions. The input variables of the network were $\mathrm{pH}$; total aerobic, yeast, mold, and coliform counts; and color analysis values measured by the machine vision system. The output variable was the storage time of the yogurt. The modeling results showed that there was excellent agreement between the experimental data and predicted values, with a high determination coefficient $\left(\mathrm{R}^{2}=0.9996\right)$ showing that the developed model was able to analyze nonlinear multivariate data with very good performance, 
fewer parameters, and shorter calculation time. The model might be an alternative method to control the expiration date of yogurt shown in labeling and provide consumers with a safer food supply [16].

The purpose of this investigation is to develop feedforward single layer models for determining shelf life of processed cheese stored at $30^{\circ} \mathrm{C}$. This study would be beneficial to cheese manufactures, consumers, regulatory authorities, academicians and food researchers.

\section{Method Material}

\subsection{Data Modeling}

Soluble nitrogen, $\mathrm{pH}$, standard plate count, yeast \& mould count, and spore count were taken as input parameters and sensory score as output parameter for developing feedforward ANN models (Fig.1). The data samples consisted of 36 observations, which were divided into two subsets, i.e., 30 were used for training the network and 6 for validating the feedforward neural network. Different combinations were tried and tested, as there is no defined rule of getting good results rather than hit and trial method. As the number of neurons increased, the training time also increased. Different algorithms were tried like Polak Fletcher Reeves update conjugate gradient algorithm, Levenberg Marquardt algorithm, Gradient Descent algorithm with adaptive learning rate, Bayesian regularization, Powell Beale restarts conjugate gradient algorithm and BFG quasi-Newton algorithm. Backpropagation algorithm based on Bayesian regularization mechanism was finally selected for training the feedforward models, as it gave better results. The network was trained upto100 epochs with single hidden layer and transfer function was tangent sigmoid while for the output layer, it was pure linear function. The Neural Network Toolbox under MATLAB software was used for development of the models.

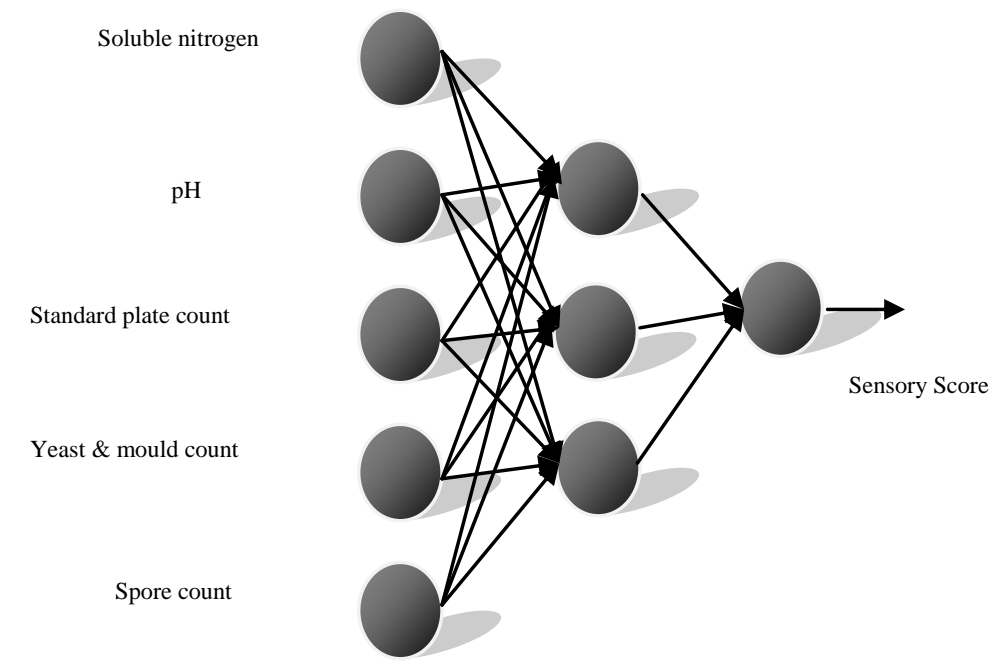

Figure1. Structure of inputs and output for feedforward network 


\subsection{Measures for Prediction Performance}

$$
\begin{aligned}
& M S E=\left[\sum_{1}^{N}\left(\frac{Q_{\text {exp }}-Q_{c a l}}{n}\right)^{2}\right] \\
& R M S E=\sqrt{\frac{1}{n}\left[\sum_{1}^{N}\left(\frac{Q_{\exp }-Q_{c a l}}{Q_{\text {exp }}}\right)^{2}\right]} \\
& R^{2}=1-\left[\sum_{1}^{N}\left(\frac{Q_{\exp }-Q_{c a l}}{Q_{\exp }^{2}}\right)^{2}\right] \\
& E^{2}=1-\left[\sum _ { 1 } ^ { N } \left(\frac{Q_{\exp }-Q_{c a l}}{\left.\left.Q_{\exp }-\overline{Q_{\exp }}\right)^{2}\right]}\right.\right.
\end{aligned}
$$

Where,

$Q_{\text {exp }}=$ Observed value;

$Q_{\text {cal }}=$ Predicted value;

$\overline{Q_{\text {exp }}}=$ Mean predicted value;

$n=$ Number of observations in dataset.

Mean Square Error: MSE (1), Root Mean Square Error: RMSE (2), Coefficient of Determination: $\mathrm{R}^{2}(3)$ and Nash - Sutcliffo Coefficient $: \mathrm{E}^{2}(4)$ were used in order to compare the prediction ability of the developed models.

\section{Results and Discussion}

Feedforward single layer model's performance matrices for predicting sensory scores are presented in Table 1. 
Table 1: Performance of feedforward single layer for predicting sensory score

\begin{tabular}{|c|c|c|c|c|}
\hline Neurons & MSE & RMSE & $\mathbf{R}^{2}$ & $\mathbf{E}^{\mathbf{2}}$ \\
\hline $\mathbf{4}$ & $1.18829 \mathrm{E}-05$ & 0.003447162 & 0.996552838 & 0.999988117 \\
\hline $\mathbf{5}$ & $1.17004 \mathrm{E}-05$ & 0.003420589 & 0.996579411 & 0.9999883 \\
\hline $\mathbf{7}$ & $1.26958 \mathrm{E}-05$ & 0.003563114 & 0.996436886 & 0.999987304 \\
\hline $\mathbf{8}$ & 0.001101823 & 0.033193726 & 0.966806274 & 0.998898177 \\
\hline $\mathbf{1 0}$ & $1.2473 \mathrm{E}-05$ & 0.00353171 & 0.99646829 & 0.999987527 \\
\hline $\mathbf{1 3}$ & 0.001812732 & 0.04257619 & 0.95742381 & 0.998187268 \\
\hline $\mathbf{1 5}$ & 0.00012198 & 0.011044445 & 0.988955555 & 0.99987802 \\
\hline $\mathbf{1 7}$ & 0.002532429 & 0.050323245 & 0.949676755 & 0.997467571 \\
\hline $\mathbf{2 0}$ & $8.78947 \mathrm{E}-05$ & 0.009375217 & 0.990624783 & 0.999912105 \\
\hline $\mathbf{2 3}$ & 0.000160044 & 0.012650866 & 0.987349134 & 0.999839956 \\
\hline $\mathbf{2 4}$ & 0.000132051 & 0.011491344 & 0.988508656 & 0.999867949 \\
\hline $\mathbf{2 5}$ & 0.000478573 & 0.021876311 & 0.978123689 & 0.999521427 \\
\hline $\mathbf{2 6}$ & 0.00049714 & 0.022296637 & 0.977703363 & 0.99950286 \\
\hline $\mathbf{2 7}$ & 0.00040249 & 0.020062142 & 0.979937858 & 0.99959751 \\
\hline $\mathbf{3 0}$ & 0.000471203 & 0.021707214 & 0.978292786 & 0.999528797 \\
\hline
\end{tabular}

Feedforward single layer models were developed for predicting shelf life of processed cheese. Single layer feedforward model with 5 neurons gave the best fit (MSE: 1.17004E-05, RMSE: 0.003420589, $\mathbf{R}^{2}$ : 0.996579411, $\mathbf{E}^{2}$ : 0.9999883), reflecting that the developed model is excellent for estimating shelf life of processed cheese stored at $30^{\circ} \mathrm{C}$ [17-22]. 


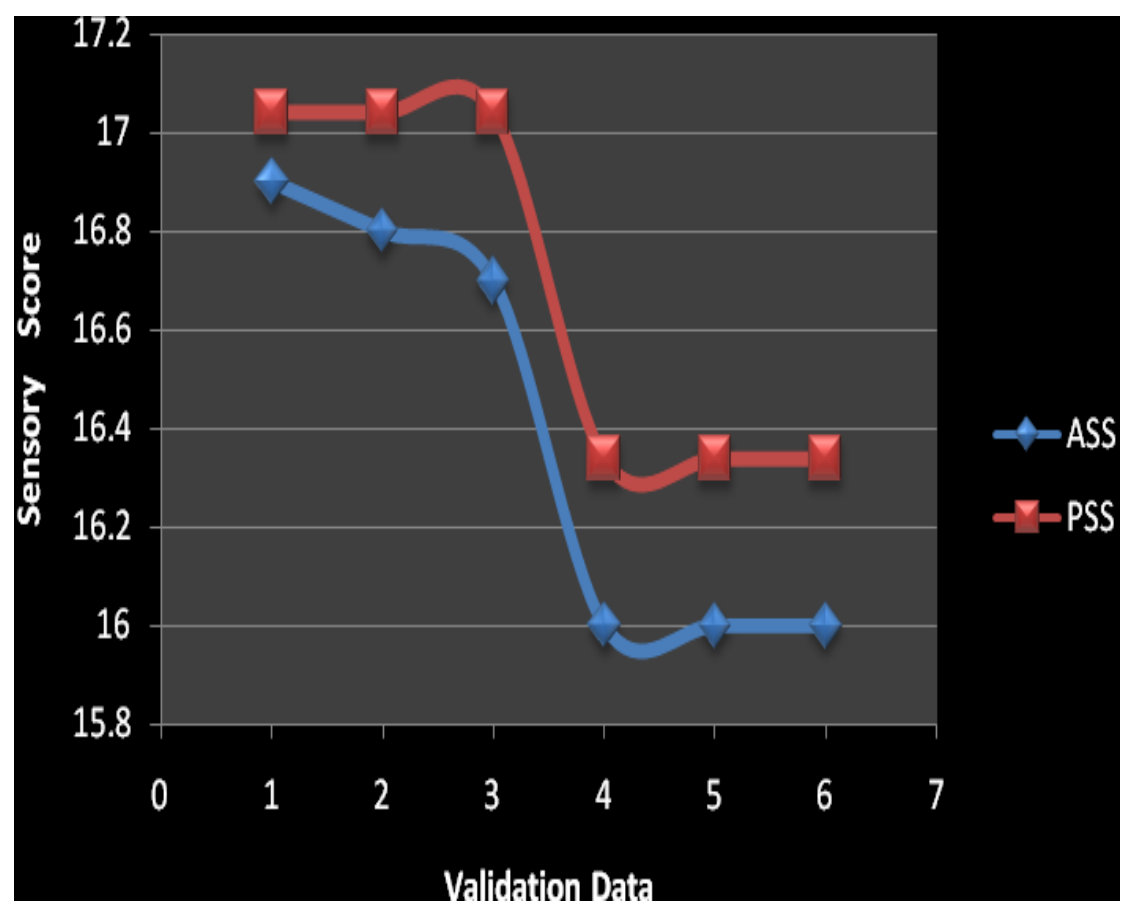

Figure 2. Comparison of ASS and PSS single layer model

\section{Conclusion}

Feedforward models single hidden layer models were developed for predicting shelf life of processed cheese stored at $30^{\circ} \mathrm{C}$. Soluble nitrogen, $\mathrm{pH}$, standard plate count, yeast \& mould count, and spore count were taken as input parameters, and sensory score as output parameter. Different combinations were tested. It was observed that single layer feedforward model with five neurons performed the best, suggesting that the developed models are able to analyze non-linear multivariate data with excellent performance, fewer parameters, and shorter calculation time. Therefore, from the investigation it can be concluded that feedforward models are good for predicting shelf life of processed cheese stored at $30^{\circ} \mathrm{C}$.

\section{References}

[1] http://www.learnartificialneuralnetworks.com/ (accessed on 30.3.2011)

[2] http://www.world-of cheese.com/history.htm (accessed on 30.3.2011)

[3] http://en.wikipedia.org/wiki/Feedforward_neural_network (accessed on 2.5.2011) 
[4] www.medlabs.com/Downloads/food_product_shelf_life_web.pdf (accessed on 1.3.2011)

[5] Sumit Goyal and G.K. Goyal. "A New Scientific Approach of Intelligent Artificial Neural Network Engineering for Predicting Shelf Life of Milky White Dessert Jeweled with Pistachio". International Journal of Scientific and Engineering Research, vol.2, no.9, (2011), pp.1-4.

[6] Sumit Goyal and G.K. Goyal. "Advanced computing research on cascade single and double hidden layers for detecting shelf life of kalakand: an artificial neural network approach". International Journal of Computer Science \& Emerging Technologies, vol.2, no.5, (2011), pp.292- 295, 2011.

[7] Sumit Goyal and G.K. Goyal. "Cascade and feedforward backpropagation artificial neural networks models for prediction of sensory quality of instant coffee flavoured sterilized drink". Canadian Journal on Artificial Intelligence, Machine Learning and Pattern Recognition.vol.2, no.6, (2011), pp.78-82.

[8] Sumit Goyal and G.K. Goyal. "Application of artificial neural engineering and regression models for forecasting shelf life of instant coffee drink". International Journal of Computer Science Issues, vol. 8, no.4, (2011), pp. 320- 324.

[9] Sumit Goyal and G.K. Goyal. "Development of intelligent computing expert system models for shelf life prediction of soft mouth melting milk cakes". International Journal of Computer Applications, vol.25,no.9, (2011), pp.4144.

[10] Sumit Goyal and G.K. Goyal. "Simulated neural network intelligent computing models for predicting shelf life of soft cakes". Global Journal of Computer Science and Technology. vol.11, no.14, version1.0, (2011), pp.2933.

[11] Sumit Goyal and G.K. Goyal. "Radial basis artificial neural network computer engineering approach for predicting shelf life of brown milk cakes decorated with almonds". International Journal of Latest Trends in Computing".vol.2, no.3, (2011), pp.434-438.

[12] Sumit Goyal and G.K. Goyal. "Brain based artificial neural network scientific computing models for shelf life prediction of cakes". Canadian Journal on Artificial Intelligence, Machine Learning and Pattern Recognition.vol.2, no.6, (2011), pp.73-77.

[13] A.G. Cruz, E.H.M. Walter, R.S. Cadena, J.A.F. Faria, H.M.A. Bolini and A.M.F. "Fileti (2009).Monitoring the authenticity of low-fat yogurts by an artificial neural network". Journal of Dairy Science, vol.92, no.10, (2009), pp.4797-4804.

[14] J. Moros, F.A. Iñón, S. Garrigues and M. Guardia, "Near-infrared diffuse reflectance spectroscopy and neural networks for measuring nutritional parameters in chocolate samples". Analytica Chimica Acta, vol. 584, no.1, (2007), pp.215-222. 
[15] Sumit Goyal and G.K. Goyal, "Radial basis (exact fit) artificial neural network technique for estimating shelf life of burfi". Advances in Computer Science and its Applications, vol.1, no.2, (2012) pp.93-96.

[16] A. Sofu and F.Y. Ekinci, "Estimation of storage time of yogurt with artificial neural network modeling". Journal of Dairy Science, vol. 90, no.7, (2007), pp.3118-3125.

[17] Sumit Goyal and G.K. Goyal, "Radial basis (exact fit) and linear layer (design) computerized ANN models for predicting shelf life of processed cheese". Computer Science Journal, 2(1), (2012), 11-18.

[18] Sumit Goyal and G.K. Goyal, "Feedforward models predicting shelf life of buffalo milk burfi". Journal of Expert Systems, 1(3), 66-70.

[19] Sumit Goyal and G.K. Goyal, "Machine learning elman technique for predicting shelf life of burfi". International Journal of Modern Education and Computer Science, 4(7), (2012), 17-23.

[20] Sumit Goyal and G.K. Goyal, "Soft computing methodology for shelf life prediction of processed cheese". International Journal of Informatics and Communication Technology, 1(1), (2012), 1-5.

[21] Sumit Goyal and G.K. Goyal, "Smart artificial intelligence computerized models for shelf life prediction of processed cheese". International Journal of Engineering and Technology, 1(3), (2012), 281-289.

[22] Sumit Goyal and G.K. Goyal, "Predicting shelf life of burfi through soft computing”. International Journal of Information Engineering and Electronic Business, 4(3), (2012), 26-33. 